\title{
Research on the Application of 3DSMAX in Animation Design
}

\author{
Dan Zhang \\ College of Arts \\ University of Science \& Technology \\ Qingdao, China \\ qdzhangdan@163.com
}

\begin{abstract}
In this paper the three-dimensional design of animation which is based on computer software is studied. The method that is used to design the animation is introduced. Through the three-dimensional design of animation, the designers can use software like 3D-Max to animate the model and to exhibit the design idea. With the construction of geometric model of stereo by the means of computer, the designers can found the model with dramatic color and texture; then can set up the model of movement and camera light. After the final rendering, which can generate a series of play, the animation of three-dimensional design can be real time continuous image. And finally realization of the computer simulation of the scene and the real object in space is accomplished. At the end of the paper, a example is made by the design software that shows the design process and effect of the three-dimensional design. The conclusion of this research is that the application of three dimension design is efficient to design the animation.
\end{abstract}

Keywords-computer animation; simulation design; three dimension; model design; geometric model

\section{INTRODUCTION}

Computer aided design is a form that is using the computer as a high-tech carrier, and is also using software to express art language and design idea. Its application is widely, such as graphic design, industrial design, environmental art design, animation design, mechanical design and other professional fields. In the environment art design, the main use of three-dimensional(3D) modeling software is to render software to complete the architectural design, landscape design, interior design, exhibition design, and furniture design, the scheme of performance and production.

In the environment art design, the design mainly refers to the two-dimensional space design or graphic design. In the software respect of view, main designers use Autodesk, which is developed by the AutoCAD company and PhotoShop, which is developed by Adobe company. Among them, AutoCAD is mainly used to draw plan, elevation, section map, and other node graph drawings.

PhotoShop is currently the more common software of image processing. With this software for drawing effect of post processing has been completed. The light and shade, saturation, texture and other can be adjusted to improve the effect of map; For 3D modeling design ,3D max or sktech up is the most typical. With it, the designers could build the model, or the generation of 3D graphics; by the software of Lightscape or Vray, the lighting rendering software, the designers can achieve the lighting and texture mapping for simulation. These are required courses for students in the university of art and design professional environment. It not only provides a hitherto unknown form of artistic expression, but the vast creative space for the designers, thus improving the working efficiency.

\section{THE SIGNIFICANCE OF THREE DIMENSIONAL COMPUTER ANIMATION}

Before the use of computer animation, all the work required is animation staff manual, 24 per second lattice picture requirements, making the number of a cartoon picture of massive[1].

Now the computer technology application greatly reduces the time of animation. The computer as a powerful tool for this art bearing animation, time, also is the new thing.

Application of computer technology in the field of animation in developed countries, the animation industry has been pushed to a new stage. In particular, the digital technology has been to the matured stage in USA. All kinds of international popular 2D, 3D and synthesis software also have everything that one expects to find, complete functions, and compatibility, suitable for running on a variety of platforms. The application of computer technology in animation matures today. Whether it is a 2D animation in Japan, or 3D animation the United States rely on the mature to make computer technology attracted the world animation enthusiasts of eyeball. In 2011 American animated film "Toy Story 3" global box office of $\$ 1063000000$, computer animation brings economic benefit is evident.

Foreign market impact put China animation into a difficult dilemma. Chinese animation has always been in a weak position in the world market, but its development potential is still underestimated. Only from the study of fundamental computer animation technology will be the new thought and technology in practical application, which our national animation can be revitalized. Combined with computer animation technology and traditional hand animation technology, it is the direction of development in the future. It obviously overcomes their shortcomings; on the contrary, the manual is unable to realize. They can learn from each other, play a complementary role[2].

My incumbency is engaged in professional animation teaching. Through the long term teaching practice, teachers have become increasingly aware of the importance of computer technology in animation application. This paper 
discusses the computer animation technology from the application point of view, combined with the actual production of the original application case, make reasonable sums up the development trend of computer animation[3].

\section{THE RELATIONSHIP BETWEEN THE TRADITIONAL ANIMATION AND COMPUTER ANIMATION}

The application of computer in the traditional twodimensional animation provides convenient and shortcut. The director Alexander Petrov, the above mentioned animation "old man and the sea", said: "The Canada Cooperative Film Bureau for me is the unprecedented excellent working environment. They gave me a set of the most advanced 'action control' (motion control) animation photography platform with computer software, the effective implementation of the number of lattice complex shooting and visual effects, but also reduce a lot of field errors caused by faults, technology is also can help art creation."

\section{A. Computer Rendering Technology}

The former has been introduced, similar to Flash, CelAction, this "full computer making" type of 2D computer animation software makes the production of $2 \mathrm{D}$ computer animation production essentially different from the traditional two-dimensional animation[4]. For example, use Animo animation is the drawn on paper drawings scanned into the computer after coloring, painting size according to the lens, or close-up is divided into three kinds, guide the animation staff will get the three lens to ensure that draw the correct role proportion. And the use of Flash, CelAction production of 2D computer animation, is directly in the computer by a proportional painting, which means that is originally a close-up lens can be easily changed or long lens camera. Background as a virtual scene, shot in the distribution of roles and props, and then based on the framework provided by the camera lens mirror frame take the location, and then began to set up the animation. From this point of view, this technique is similar to the traditional stop motion animation photography, and the two-dimensional "unlike traditional graphic animation[5].

Three dimensional computer animation omitted handmade model of traditional puppet animation step by step, the direct use of computer 3D animation software is to create animation role in the computer animation scene model, equivalent to complete the traditional stop motion animation in computer. The application of computer animation brings convenience and shortcut to the traditional stop motion animation, but also give traditional animation has a great impact.

As one of the famous British animation company Aardman founder Peter Rod (Peter Lord) has directed "chicken run", "Wallace and Gromit demons rabbit curse" and many other animation. In talking about the animation will be replaced by computer technology, he said: "twenty years ago, some images can only be used in stop motion animation, now is different. For example, Ray Halihausen (Ray Harryhausen) film, the only way to create lifelike monsters and dinosaurs is stop motion animation. Such as
"Star Wars" the first set and the second set, to create walking in the frozen planet graphic monster, the only way is to stop motion animation. "The future soldier" in the first episode, characters come out from the crash of the burning truck, thus it is not made out of stop motion animation. Of time, this is the only can create these image method. Therefore fixed at that time in the movie industry occupies a place. Now these are being replaced by computer technology, before the example can be used to produce computer technology." Thus, computer animation is created a huge impact to the traditional stop motion animation[6].

\section{B. Computer Animation Technology}

Computer technology in all aspects of stop motion animation provides powerful help, in Gromit, the rabbit monster we have thirty plastic clay for the rabbit, also have the computer film making rabbit, of which there are very practical considerations. A movie is floating in the space of three degrees, with fixed word of the shooting is very difficult, very expensive and delicate work, and using computer image to make words is relatively simple. With digital later, can easily freeze frame shot role to lift from the ground, to make them fly, and synthesis is also very easy to do. The foreground and background can be respectively to film synthesis, and a background filming frames can also be synthetic, and it is very practical. Ten years ago we spent nine great lengths, after a lot of difficulties to make the audience feel fly effect. But now these effects in digital mapping is a common sense, it is easy to do. Now in our studio, we sometimes feel studio space is too small, do not make a big scene lens we want, or some more momentum approach. In synthesis, these ideas can be more easily achieved. We do the same scene in the studio downstairs, we also do a copy scene three to four times as much, because we still want to use the camera make smooth reading panoramic. But if we can put the foreground and background photographed separately and then the computer synthesis, then we do not need to set up so many times and so many scenarios, this can save a lot of budget and space, this is a very practical method[7].

But in the affirmation of computer technology as a stop motion animation to provide convenient, Adelman said: "make computer animated people let the movie looks smooth, perfect, dazzling, making animation relative will go to emphasize simple manual texture, that there is a kind of spirit, and people like this kind of spirit." Thus, stop motion animation will be replaced by $3 \mathrm{D}$ computer animation technology, just as the end of nineteenth Century painters are worried about whether the photography appearance will replace the painting in general, the historical development of proof painting did not disappear, just unloaded a realistic task of photography and pay more attention to explore the essence of painting, and then go elsewhere for unique painting effect of [8].

Nowadays in computer animation, the drawing board by using the computer software connection configuration with pressure sensing function, make the painting can be like in the ordinary paper, painted with thickness and shade change lines, to create with hand drawn animated line strokes feeling computer animation is a cartoon very popular means of production[9]. 
Combined with computer animation technology and traditional hand animation technology, is one of the direction of development in the future. It obviously overcomes their shortcomings, is a computer can not do, hand can do; on the contrary, the manual is unable to realize, can realize the computer. They can learn from each other, play a complementary role[10].

\section{THE COMPUTER 3D ANIMATION SOFTWARE}

The producer of the Autodesk 3ds Max is easy to operate, and with other relevant software with smooth. 3DS Max has a wide variety of plugins, which makes the software function greatly complement and improve. Some features is incorporated into the 3DS Max, such as CS (Character Studio) which is first used to quickly establish a human body model of the 3DS Max plugin. Science the 3DS Max 4 version CS is incorporated into the software, become a function module of 3DS Max. And as the German MentalImage company produced, rendering engine high performance MentalRay, 3DS Max MentalRay can make up for the disadvantages of its external weak performance, from the 3DS Max 6 version MentalRay is integrated into the 3DS Max software[11].

With many modules and tools of 3DS Max shows many excellent functional properties in the production of computer 3D animation:

Interface

The modeling, animation, rendering settings synthesis in the same interface, and can be custom interface layout and shortcut, convenient operation.

Modeling

Modeling of flexible and diverse way, basic geometry modeling software provides more convenient, NURBS tool to make more accurate, realistic modeling.

Animation

You can animate any object, make accords with the rule of natural movement, the particle system can generate advanced animation effect, the CS module to enhance the creation of animation role function.

Lighting and camera

Provide realistic effect of various conventional virtual light, heat, light, glare, camera can set along path to simulate the real lens to do all kinds of sports shooting effect.

Rendering performance

Can be connected to realize the high efficiency, high quality through the multi-thread and the network rendering, animation picture control effect by ray tracing effect can be generated film quality level resolution up to 10000 lines.

Development tools

Animators can according to their own needs, the use of advanced MAXScript animation programming language developed feature or plug-in.

Scalability

3DS Max not only has various kinds of plug-ins, can also through the application of combination of Edit, Paint, Effect, Combustion and other software components, in 3D animation, graphic image processing, nonlinear editing and special effects production in an animation system, used to complete the tasks of computer 3D animation.

\section{EXAMPLE OF COMPUTER 3D ANIMATION}

Making process of this chapter to $3 \mathrm{D}$ computer animation the adventures of "stars" as an example, detailed application of animation company domestic computer 3D animation technology in actual animation in the project.

\section{A. Character Design}

"The adventures of the stars" is a domestic original adventure inspirational animated feature, currently making the first three episodes have been completed. The hero star into the world is the magic world, there are many interesting islands: sugar ball Island, island mushroom, toothpaste, flowers, jeans Island Island island...... He will go together with new friends and adventure, in the exploration process of stars encountered all sorts of Wonderland, wonderful story, full of touching fairy tale color.

Design of scene model scene plays a major role in the 3D model project, need according to the pre-set to create different 3D scene in 3D software, such as the "Star" in the adventures of candy Island, island mushroom etc..

The main story unfolds in the magical world, so we will focus primarily on the magic scene, different scenarios need to reflect the different style[12].

The setting of the story is mostly are relatively large, doing the scene should fully show the key factors in each scene, also want to pay attention to streamline the degree model.

In the scene model making to ensure certain subdivision level based on minimizing the number of faces, so that after the texture size was relatively small, is to reduce the burden of rendering, some details of the model can be used to make up the texture.

Texture mapping:

Material work has a vital role in the production link, and UV are the basic requirements to do texture mapping. We in the "Star" in the adventures of two parts: the role of material texture, scene material.

The role of textures - the protagonists of the material is relatively high, the goal is to achieve better rendering effect, the rest of the supporting material according to the fine degree appearance rate and whether there is a close-up of reasonable distribution of materials.

\section{B. Character Design}

According to the character, before we collected some children clothing for reference on the internet, the piece appears the role in color follow the bright and lively reflect children's natural and healthy skin.

Each of the major role of using three sets of maps, respectively is: the diffuse color, speculation, and bump mapping[13]. 

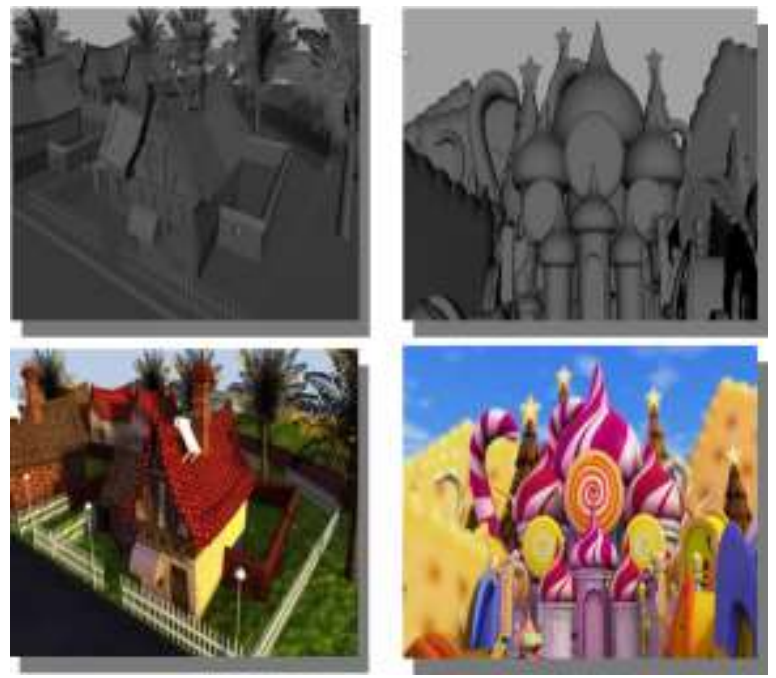

Figure 1. View Design Example

The scene model material - the story is in the human society and the occurrence of magic in the world, so how to distinguish between reality and fantasy is a basic target. According to the different scene textures in different atmosphere, such as sing in the color residence reflects the warm, and in the candy world color is very colorful, reflecting the candy to the person's appetite[14].

\section{CONCLUSION}

Nowadays in computer animation, the drawing board by using the computer software is connected with pressure sensing function, which makes the painting can be like the ordinary paper that painted with thick and thin, shade change lines, to create with hand drawn animated line strokes feeling. Computer animation is a cartoon very popular means of production.

Combined with computer animation technology and traditional hand animation technology is the direction of future development. It obviously overcomes their shortcomings, which is a computer can not do but hand can do; on the contrary, the manual is unable to realize, nor can realize the computer. They can learn from each other, and play a complementary role.

\section{ACKNOWLEDGMENT}

This paper is supported by the 2013 Key Culture Project of Shandong Province Department of Culture: Study on the Mobility of Architecture, No: 2014220.

This paper is supported by the 2014 Key Culture Project of Shandong Province Department of Culture: The Study of Residential Environment Based on the Old Aging Society, No:2013373.

This paper is supported by the 2011 Key Culture Project of Shandong Province Department of Culture: Research on Regional Planning City Neighborhood Characteristics, No: 2011176.

This paper is supported by the 2011 Key Culture Project of Qingdao University of Science\&Technology, Study on the Elderly Living Building, No:11xc01.

This paper is supported by the 2011 Key Culture Project of Qingdao University of Science\&Technology,
Research on the Design of Public Facilities Based on the Characteristics of Regional Culture, No:10xc32.

\section{REFERENCES}

[1] Jen-Hui Chuang, Chi-Hao Tsai, Min-Chi Ko, "Skeletonization of Three-Dimensional Object Using Generalized Potential Field", IEEE Transactions on Pattern Analysis \& Machine Intelligence, vol.22, no. 11, pp. 1241-1251, November 2000, doi:10.1109/34.888709

[2] Xia Ning, Li Aishuang, Su Zhu-hua, Gan Yangying, Niu Xiaoguo, Technique of virtual plant growth and its applications prospect to agriculture, Guangdong Agricultural Sciences;2009-09

[3] Xu Ying, Improvement of Mesh-based Image Morphing Technique, Journal of Mianyang Normal University;2008-05

[4] XIONG Zhen-qi,NING Ru-xin,LIU Jian-hua,LIU Wei-dong (School of Mechanical and Vehicular Engineering,Beijing Institute of Technology,Beijing 100081,China);Pre-analysis of Connecting Precision of Missile Sections in Virtual Environment[J];Acta Armament;2008-08

[5] YAN Yun-hui, ZHANG Hai-yan, YANG Hui-lin, LI Chengfeng(School of Mechanical Engineering \& Automation, Northeastern University, Shenyang 110004, China. Correspondent: YAN Yun-hui, professor, E-mail: yanyh @ mail.neu.edu.cn);Modeling, Interference Checking and Simulation of Virtual Assembly Systems[J];JOURNAL OF NORTHEASTERN UNIVERSITY;2004-02

[6] Zhou Minghua and Wang Guozhao(Institute of Computer Graphics and Image Processing, Department of Mathematics, Zhejiang University, Hangzhou 310027)(Department of Applied Mathematics , Zhejiang University of Technology, Hangzhou 310032); Genetic Algorithm-Based Least Square Fitting of BSpline and Bezier Curves[J];Journal of Computer Research and Development;2005-01

[7] Xie, J., Chen, Y., Liu, J., Miao, C., Gao, X.: Interactive 3D caricature generation based on double sampling. In: ACM International Conference on Multimedia, Vancouver, British, Columbia, Canada, pp. 745-748 (2009)

[8] Zhang, M., Liu, S., Wang, J., Shen, H., Pan, Z.: The 3D caricature face modeling based on aesthetic formulae. In: International Conference on Virtual Reality Continuum and Its Applications in Industry, COEX, Seoul Korea (2010)

[9] Clarke, L., Chen, M., Mora, B.: Automatic generation of 3D caricatures based on artistic deformation styles. IEEE Trans. Vis. Comput. Graph. 17(6), 808-821 (2011)

[10] Zhang, M., Liu, S., Wang, J., Shen, H., Pan, Z.: The 3D caricature face modeling based on aesthetic formulae. In: International Conference on Virtual Reality Continuum and Its Applications in Industry, COEX, Seoul Korea (2010)

[11] Fu, G., Chen, Y., Liu, J., Zhou, J., Li, P.: Interactive expressive 3D caricatures design. In: IEEE International Conference on Multimedia and Expo (ICME), Hannover, Germany, pp. 965 - 968 (2008)

[12] Xie, J., Chen, Y., Liu, J., Miao, C., Gao, X.: Interactive 3D caricature generation based on double sampling. In: ACM International Conference on Multimedia, Vancouver, British, Columbia, Canada, pp. 745 - 748 (2009)

[13] Ersotelos, N., Dong, F.: Building highly realistic facial modeling and animation: a survey. Vis. Comput. 24(1), 13 - 30 (2008)

[14] Fujiwara, T., Koshimizu, H., Fujimura, K., Fujita, G., Noguchi, Y., Ishikawa, N.: A method for 3D face modeling and caricatured figure generation. In: IEEE International Conference on Multimedia and Expo (ICME), Lausanne, Switzerland, pp. 137 140 (2002) 